\title{
BMJ Open Quality Use of audit, feedback and education increased guideline implementation in a multidisciplinary stroke unit
}

\author{
Angela Vratsistas-Curto, ${ }^{1}$ Annie McCluskey, ${ }^{2,3}$ Karl Schurr ${ }^{3}$
}

To cite: Vratsistas-Curto A, McCluskey A, Schurr K. Use of audit, feedback and education increased guideline implementation in a multidisciplinary stroke unit.BMJ Open Quality 2017;6:e000212. doi:10.1136/ bmjoq-2017-000212

- Additional material is published online only. To view, please visit the journal online (http://dx.doi.org/10.1136/ bmjoq-2017-000212).

Received 26 September 2017 Revised 4 October 2017 Accepted 4 October 2017

\section{CrossMark}

${ }^{1}$ Musculoskeletal Health Sydney, School of Public Health, The University of Sydney, New South Wales, Australia

${ }^{2}$ Discipline of Occupational Therapy, Faculty of Health Sciences, University of Sydney, Lidcome, New South Wales, Australia

${ }^{3}$ The StrokeEd Collaboration, Regents Park, New South Wales, Australia

Correspondence to Ms Angela Vratsistas-Curto, Musculoskeletal Health Sydney, School of Public Health, The University of Sydney, New South Wales, Australia; angela.vratsistas@sydney. edu.au

\section{ABSTRACT}

Background The audit-feedback cycle is a behaviour change intervention used to reduce evidence-practice gaps. In this study, repeat audits, feedback, education and training were used to change practice and increase compliance with Australian guideline recommendations for stroke rehabilitation.

Objective To increase the proportion of patients with stroke receiving best practice screening, assessment and treatment.

Methods A before-and-after study design was used. Data were collected from medical records ( $n=15$ files per audit). Four audits were conducted between 2009 and 2013. Consecutive files of patients with stroke admitted to the stroke unit were selected and audited retrospectively. Staff behaviour change interventions included four cycles of audit feedback, and education to assist staff with change. The primary outcome measure was the proportion of eligible patients receiving best practice against target behaviours, based on audit data.

Results Between the first and fourth audit (2009 and 2013), 20 of the 27 areas targeted (74\%) met or exceeded the minimum target of $10 \%$ change. Practice areas that showed the most change included sensation screening $(+75 \%)$ and rehabilitation $(+100 \%)$; neglect screening (+92\%) and assessment (100\%). Some target behaviours showed a drop in compliance such as anxiety and depression screening $(-27 \%)$ or little or no overall improvement such as patient education about stroke $(6 \%$ change).

Conclusions Audit feedback and education increased the proportion of inpatients with stroke receiving best practice rehabilitation in some, but not all practice areas. An ongoing process of quality improvement is needed to help sustain these improvements.

\section{INTRODUCTION}

Translating research into practice from clinical guidelines is one of the most challenging stages of evidence-based practice. A recent systematic review suggests that tailored interventions which target identified determinants of practice can be effective in producing small-to-moderate amounts of practice change. ${ }^{1}$ Tailored interventions are planned strategies for improving practice that prospectively target identified determinants of practice, such as knowledge and skill barriers. ${ }^{1}$ Interventions used are often multifaceted and can include printed educational materials (PEMs) (e.g., guidelines, journal articles) ${ }^{2}$; outreach visits (e.g., group or $\left.1: 1\right)^{3}$; group education (e.g., lectures, workshops or facilitated interactive group discussions) ${ }^{4}$; opinion leaders ${ }^{5}$ and audit and feedback. ${ }^{6}$

\section{Audit and feedback}

Systematic reviews have shown that cycles of audit, with written and verbal feedback can change clinical practice and nominated behaviours by small to modest amounts (median change of $1 \%-6 \%){ }^{6-8}$ Audit of medical records has been used to change the behaviour of stroke unit teams in the UK, ${ }^{9}$ the Netherlands, ${ }^{10}$ Australia, ${ }^{11}$ Spain $^{12}$ and Canada. ${ }^{13}$ To date, most studies have focused on practice areas related to emergency and acute stroke care. Audits of rehabilitation care are needed to determine the provision of optimal interventions for stroke survivors and are relevant to allied health clinicians.

Guideline recommendations are used as the basis for most of these clinical audits. The Stroke Foundation in Australia coordinates an audit of acute stroke services every 2 years, with criteria derived from the National Stroke guidelines. ${ }^{14} 15$ Australian national audits began to focus on rehabilitation practice in $2011 .^{16}$ Prior to 2011, feedback was only provided about acute care and selected rehabilitation criteria such as allied health assessment and patient education. ${ }^{17} 18$ In 2009 , our stroke service began internal audits to investigate compliance with stroke rehabilitation guideline recommendations.

\section{Education interventions}

Dissemination of PEMs such as clinical guidelines and journal articles is widely used as an implementation intervention. While dissemination is a relatively passive intervention, PEMs do change clinical practice by small amounts (mean change of 13\%) when compared with no intervention. ${ }^{2}$ Group education such as lectures, workshops or facilitated interactive group discussions is 
another practice change intervention commonly used in implementation research. When used alone or combined with other interventions, group education has also been shown to result in small improvements in professional practice $(6 \%-10 \%){ }^{4}$

\section{Identifying determinants of practice}

Identifying and addressing the determinants of practice may also influence the effectiveness of tailored interventions used to improve practice. Determinants of practice (also known as barriers and enablers) are factors that can either prevent and/or enable improvements. ${ }^{19}$ In a concurrent qualitative study, we conducted focus group interviews to identify determinants of practice following initial audit feedback. ${ }^{20}$

\section{Objective}

To increase the proportion of patients with stroke receiving best practice screening, assessment and treatment.

\section{METHODS \\ Design}

A before-and-after study design was used. The implementation process included four components: four cycles of audit and feedback, identifying determinants of practice, provision of educational materials and group education sessions to assist staff with change. Table 1 gives an overview of the implementation process and the stages. Ethical approval was obtained from the local health district to conduct the study (Ref No. QA 2008/046). Approval was granted to audit patient records without gaining written consent from individual staff, or from patients who had been discharged. The TIDieR ${ }^{21}$ and SQUIRE guidelines ${ }^{22}$ were used for reporting the current audit and feedback study.

\section{Setting}

The project was conducted at one metropolitan stroke unit in Sydney, Australia. This is a 20-bed comprehensive unit where patients are admitted directly from the emergency department to receive their acute care and remain in the unit for their inpatient rehabilitation. ${ }^{23}$

\section{Participants}

A total of 31 stroke unit staff across eight disciplines (medical, nursing, occupational therapy, physiotherapy, speech pathology, social work, dietetics and orthoptics) were invited to participate in the study. Staff were notified of our intention to audit their written medical records and to provide subsequent feedback. No patients participated in the study.

\section{Baseline audit}

The audit checklist

An audit checklist was developed in 2009, which included recommendations from Australian (2005), Scottish, English and Canadian stroke guidelines ${ }^{1424-26}$ for the initial audit. The checklist was updated using 2010 Australian guidelines for subsequent audits. ${ }^{15}$ Practice areas audited included communication, swallowing, upper limb function, mobility, activities of daily living, vision, neglect, return to work, depression/anxiety, sexuality, driving, task practice, education and home assessment. Audit criteria were rated using yes/no/not applicable response options. Questions were asked about screening, assessments conducted and intervention provided in relation to each practice area. A sample of the audit tool is provided in online supplementary 2.

The baseline audit (audit 1) was conducted in November 2009. For each audit, a consecutive, retrospective sample of 15 medical files was selected of patients with stroke admitted to the stroke unit. When a file was not available for audit, the previous patient name on the list was selected.

Audits were conducted by allied health and nursing staff, students and members of the research team. Multiple auditors $(n=15)$ were used to raise staff awareness of the auditing process, individual and team practice and to engage staff in the audits. Each staff member was asked to audit at least one medical record. Two medical files from each phase of audits were double audited and

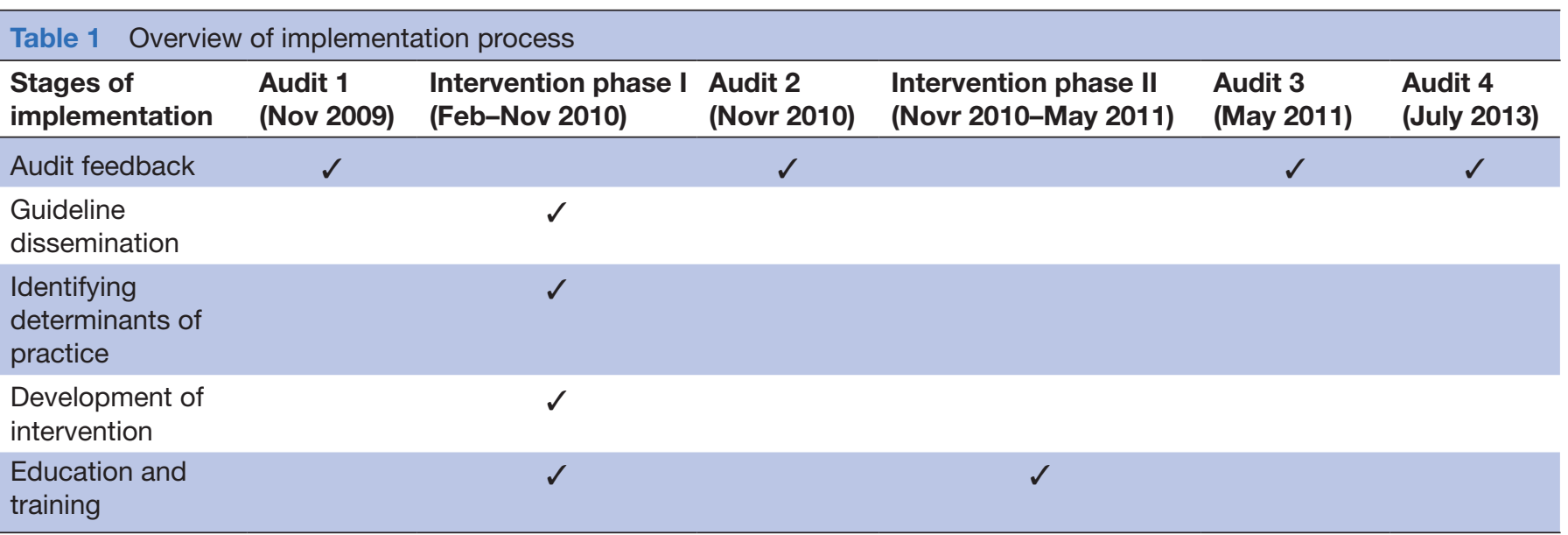


coded by one of the two research team members to check for rater consistency. Differences were discussed and consensus reached when necessary. No formal study of rater agreement was conducted.

\section{Audit feedback and guideline dissemination}

After the baseline audit, the research project manager (RPM) presented the results to allied health, nursing and medical staff about each profession's compliance with the relevant criteria, in a face-to-face feedback session. Presentations with graphs and tables were used to present results for each practice area (e.g., education), and summarise individual practice areas with good compliance or areas needing improvement (see online supplementary 3 for an example of audit feedback presented to staff). An audit summary report was provided to all staff describing the audit results. Reports included a summary of individual practice areas with good compliance and those areas needing improvement. For each practice area (e.g., communication), results were displayed in tables showing the $\%$ compliance for each criterion audited (see online supplementary 4 for an example of a feedback report). Each discipline was then invited to nominate at least one area for practice improvement. The RPM provided each discipline with a printed copy of the guidelines ${ }^{14}$ and highlighted recommendations relevant to them and the nominated areas. The RPM provided feedback sessions and an audit summary report to all staff after each subsequent audit.

\section{Identifying determinants of practice}

Assessing the problem and linking to Theoretical Domains Framework (TDF)

A barrier analysis was conducted by the researchers in 2009 after the initial audit. To identify barriers, we used two methods which are recommended when conducting implementation research. ${ }^{27}$ We conducted focus group interviews between July and October 2010 (described elsewhere ${ }^{20}$ ) with allied health, nursing and medical staff which were transcribed and analysed for themes using the $\mathrm{TDF}^{28}$ This parallel qualitative study was approved as a separate project (ref: 2009/012). The TDF comprises 14 domains and factors that are likely to influence healthcare professional behaviour and can be targeted to facilitate practice change: knowledge; skills; social/professional role and identity; beliefs about capabilities; optimism; beliefs about consequences; reinforcement; intentions; goals; memory, attention and decision processes; environmental context and resources; social influences; emotion and behavioural regulation. ${ }^{28}$

Six key barriers were identified and were different for each discipline and intervention. (1) Beliefs about the capabilities of individual clinicians or their discipline to deliver an assessment or intervention, and beliefs about patients' ability to participate in an intervention. For example, physiotherapists (PTs) had concerns about the physical demands on staff and patients during treadmill training with weight support. (2) Beliefs about the consequences, both positive and negative, of providing or not providing an assessment or intervention was the second category of barrier. For example, speech pathologists (SPs) were concerned about incorrectly interpreting results of aphasia assessments for patients with limited English and whether vision problems would alter test scores. (3) Memory attention and decision processes was a third category of barrier and refers to systems and prompts that reminded clinicians to deliver an intervention. For example, medical staff reported a lack of systems that prompted discussion and documentation regarding return to work, driving and anxiety/depression. (4) Limited knowledge and skills required to deliver evidencebased assessment and intervention was another category of barrier. For example, occupational therapists (OTs) lacked knowledge and skills in assessment and interventions for managing sensation and neglect. (5) Intention and goals was the fourth category of barrier and refers to clinicians' lack of intention to provide assessment/ intervention and having/prioritising goals. For example, OTs reported difficulty completing sensation and neglect assessment and intervention due to competing rehabilitation/goal priorities. (6) Limited resources was the final category of barrier. For example, nursing staff lacked access to educational materials (including translated material) to give to patients. Further details of barriers are provided in online supplementary 1 and reported elsewhere. ${ }^{20}$

\section{Developing the intervention}

The researchers used the behaviour change wheel (BCW) to design the intervention. ${ }^{29}$ The online supplementary 1 shows how the TDF and BCW were applied to targeted practice areas, linking the barriers and enablers to the TDF, BCW domains, intervention functions (IF) and resulting intervention.

\section{Mapping the BCW Capability-Opportunity-Motivation-}

Behaviour (COM-B) system to TDF domains

using the BCW, the TDF domains were mapped to the COM-B domains. The process assumes that capability, opportunity and motivation interact to generate behaviour change. ${ }^{29}$ For example, in order to improve the OTs' knowledge and skills in neglect rehabilitation, we needed to maximise their psychological capability.

Linking components of the COM-B system to relevant intervention functions: the BCW links COM-B and TDF domains to IF. This linkage process helped to identify intervention options that were most likely to address key barriers identified by each discipline. Using the above example, if knowledge is an identified barrier, education is the recommended IF.

\section{Forming possible solutions (behaviour change techniques (BCTs))}

The researchers then selected interventions to be implemented with each discipline and for each practice area, based on relevance, feasibility and acceptability of the intervention. 


\section{Education and training sessions}

During phase I of the intervention period (FebruaryNovember 2010), allied health, nursing and medical staff met with the RPMs monthly and participated in education and training sessions. During phase II (November 2010May 2011), training sessions were reduced to bimonthly/ quarterly. Compliance targets were set and progress was also reviewed during the sessions.

Several different BCTs were used with each discipline during the education/training sessions to improve their practice. Within the domain/IF of education, two main BCTs were used. First, feedback on behaviour was provided to all disciplines following medical record audits about their compliance with delivery of screening/ assessments and interventions. Second, information was provided about the health consequences and outcomes of performing a behaviour. For example, using published research to provide OTs with information about the benefits and outcomes of delivering interventions for sensation and neglect.

Within the IF of training, three key BCTs were used, including demonstration, instruction and practice of a behaviour. For example, physiotherapy staff participated in a training session where senior staff demonstrated and staff practised skills required for training sitting balance and treadmill training. Occupational therapy staff participated in training about neglect assessment and rehabilitation with a known clinical expert. Joint neglect rehabilitation sessions were also conducted with the stroke unit orthoptist.

Within the IFs of environmental restructuring and enablement, two key BCTs were used. First, prompts and cues were provided, such as regular caseload reviews with PTs to prompt provision of sitting balance and treadmill training. A case conference checklist was introduced for use by medical staff to prompt action regarding anxiety and depression, driving and return to work. Second, resources (objects) were ordered and added to the environment/setting to assist with screening and assessment e.g. the Nottingham Sensory Assessment (OT) and Mississippi Aphasia Screening Tool (SP). Other materials introduced to the environment included education packs and an ink stamp for documentation of education.

Within the IF of persuasion, three primary BCTs were used. First, information was provided about the health consequences and outcomes as described earlier. Second, the use of visual and verbal communication from credible sources to encourage clinicians to use assessments or interventions. For example, use of supporting published research in discussions with OTs and PTs and an expert neglect clinician and orthoptist with OTs to encourage increased use of interventions. Third, encouraging staff to focus on past success with implementing assessments or intervention. For example, encouraging physiotherapy staff to reflect on and discuss previous success with delivering sitting balance and treadmill training to patients.

\section{Evaluation}

Subsequent audits were conducted in November 2010 (audit 2), May 2011 (audit 3) and July 2013 (audit 4) to measure change using the same methods as the baseline audit. A total of 60 medical files ( $\mathrm{n}=15$ per audit) were audited over a 4-year period. The primary outcome measure was the proportion of eligible patients receiving best practice against target behaviours, based on audit data. Based on the reviews by Grimshaw et $a l^{7}$ and Jamtvedt $e t a l,{ }^{8}$ consensus was reached that a $10 \%$ change in the target practice behaviours would be the goal for each practice area (i.e., the predetermined minimum clinically worthwhile difference).

\section{RESULTS}

\section{Sample characteristics}

Stroke unit staff participant characteristics

A total of 26 staff and students from the stroke unit audited at least one record. The majority of auditors were female $(n=21,81 \%)$. Disciplines included OTs $(n=7,27 \%)$ PTs $(n=6,23 \%)$, students $(n=5,19 \%)$, SPs $(n=2,8 \%)$ and nurses $(n=2,8 \%)$ as well as researchers $(n=4,15 \%)$.

\section{Patient participant characteristics}

In the 2009 audit $(n=15)$, the mean patient age was 73 years $(\mathrm{SD}=13.3)$, and $47 \%$ were male. For the 2010 audit, the mean patient age was 75 years $(\mathrm{SD}=12.8)$, and $60 \%$ were male. For the 2011 audit, the mean age was 78 years $(\mathrm{SD}=9.4)$, and $47 \%$ were male. For the 2013 sample, the mean age was 77 years $(\mathrm{SD}=7.9)$, and $67 \%$ were male. There was no significant difference in patient age or gender across the samples.

\section{Baseline results}

The baseline audit of medical records revealed a number of practice areas to be improved. Staff agreed that compliance of $60 \%$ or less indicated areas requiring practice improvement. Compliance in the following areas was low and became the target for practice improvement: management of communication $(0 \%-53 \%$ of eligible files complied); treadmill training (17\%), sitting balance training $(25 \%)$, management of upper limb sensation $(0 \%)$; management of neglect $(0 \%-53 \%)$; patient and carer education $(0 \%-27 \%)$; anxiety and depression screening $(27 \%)$ and discussion regarding return work and driving $(0 \%-33 \%)$ (see table 2 for detailed baseline results).

\section{Audit results}

Table 2 and figure 1 present results over time for each discipline and practice area targeted.

\section{Speech pathology}

After the final audit in 2013, overall improvements of between $5 \%$ and $100 \%$ were noted in the management of communication and swallowing. For dysphagia screening, a small overall improvement of $5 \%$ was reported from $80 \%$ (95\% CI $60 \%$ to $100 \%$ ) in 2009 , up to $85 \%$ (95\% CI 


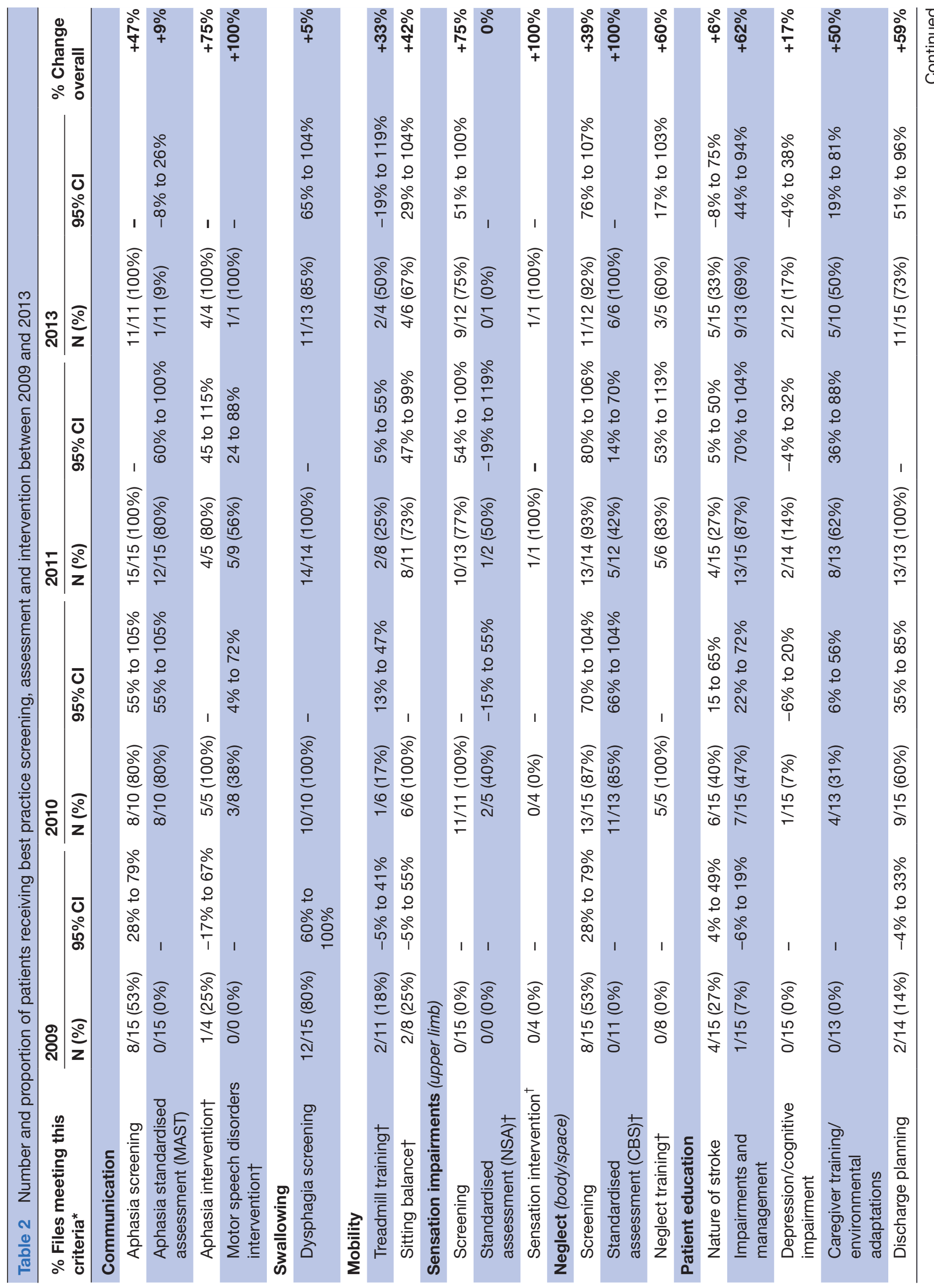




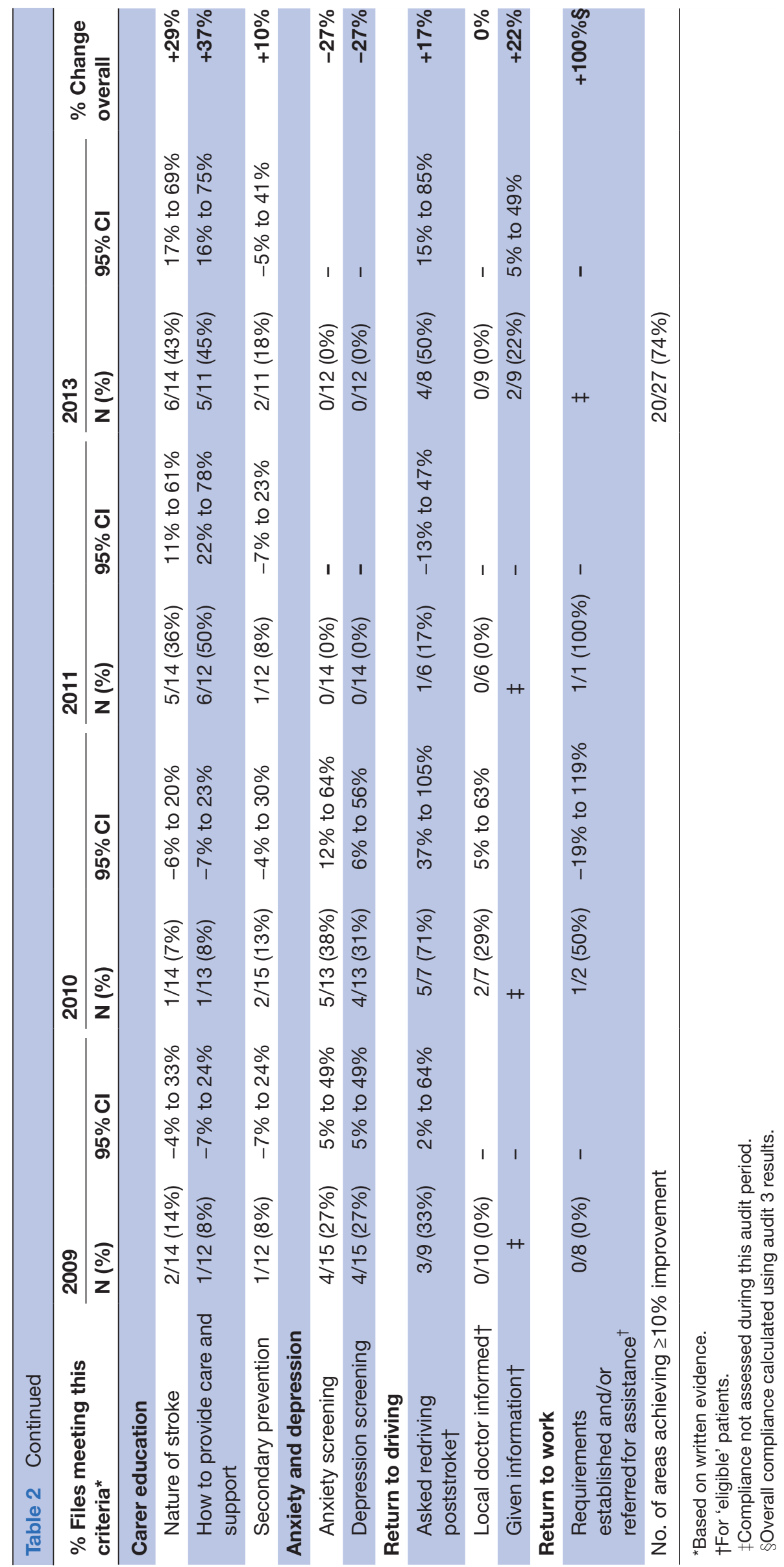


A: Changes in management of aphasia, motor speech disorders and swallowing

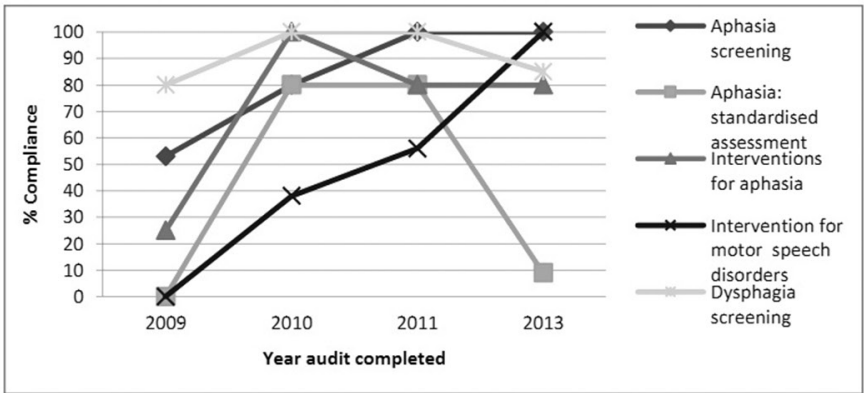

B: Changes in provision of treadmill and sitting balance

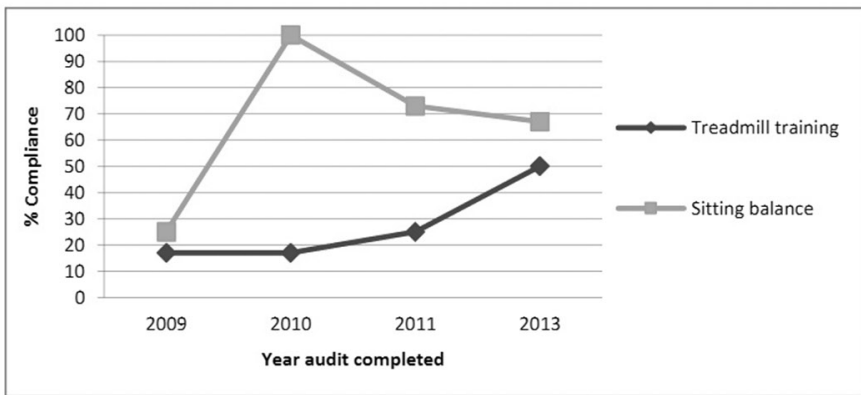

C: Changes in the assessment and management of sensation

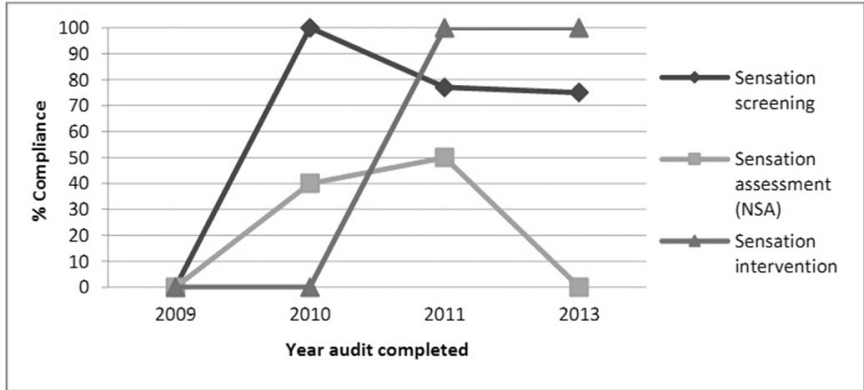

\section{D: Changes in management of neglect}

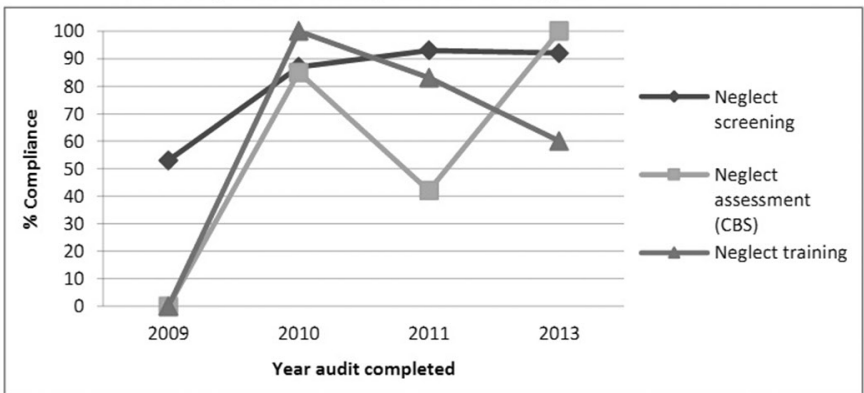

E: Changes in patient and carer education

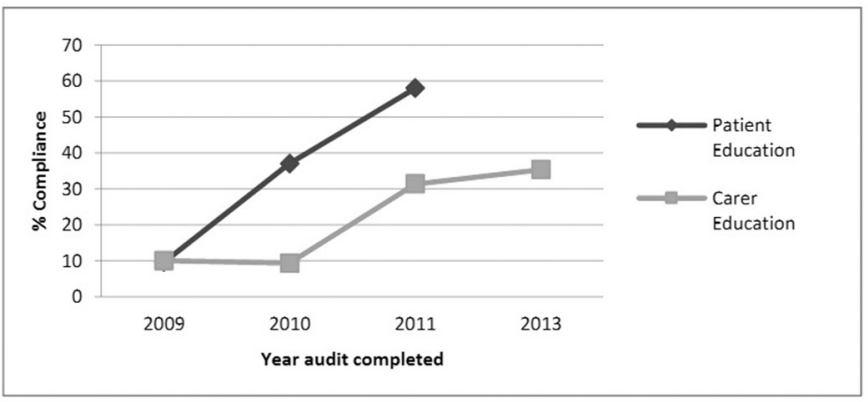

F: Changes in screening of anxiety and depression

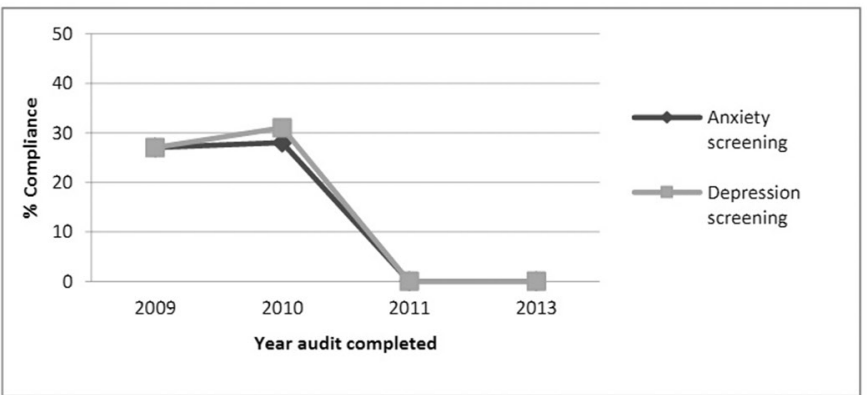

G: Changes in management of return to driving

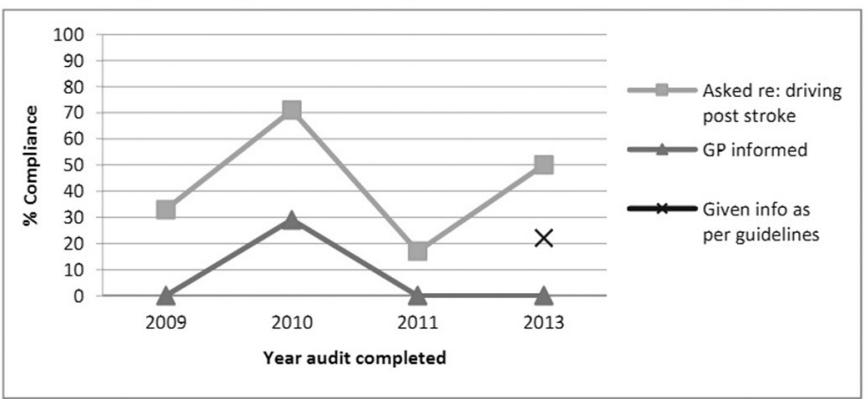

$\mathrm{H}$ : Changes in management of return to work

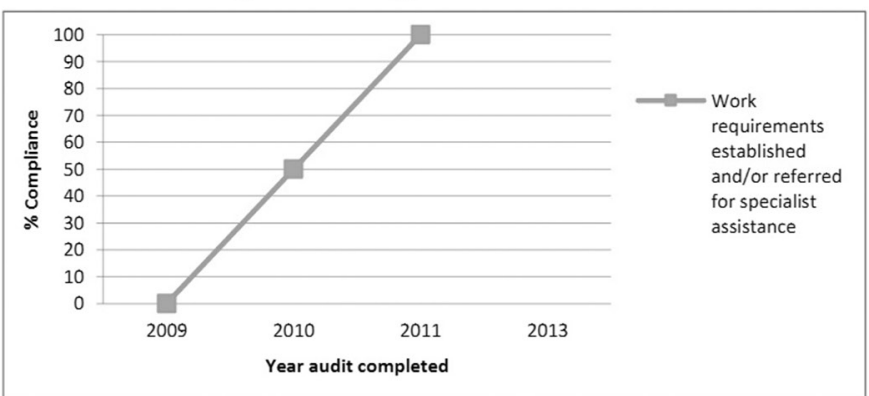

Figure 1 Proportion of patients receiving best practice screening, assessment and intervention between 2009 and 2013.

$65 \%$ to $104 \%$ ) in 2013. A large change was recorded in motor speech intervention from $0 \%$ in 2009 , up to $100 \%$ in 2013.

\section{Physiotherapy}

Overall, moderate improvements in the delivery of treadmill training with partial body weight support and sitting balance training practice were noted. An overall improvement of $33 \%$ was recorded in the provision of treadmill training, from $18 \%$ (95\% CI $-5 \%$ to $41 \%$ ) in 2009 up to $50 \%$ (95\% CI $-19 \%$ to $119 \%$ ) in 2013 . Provision of sitting balance training improved from $25 \%(95 \% \mathrm{CI}-5 \%$ to $55 \%)$ to $67 \%$ (95\% CI $29 \%$ to $104 \%$ ), an overall improvement of $42 \%$.

Occupational therapy

Overall improvements of between $0 \%$ and $100 \%$ were noted in management of upper limb sensation and neglect impairments. While there was some improvement 
noted after the second and third audits, no overall change $(0 \%)$ was seen in the standardised assessment of sensation with no patients being assessed at baseline in 2009 or after the final audit in 2013. A large change was recorded in provision of sensory training from no patients receiving the intervention at baseline $(0 \%)$ to all eligible patients (100\%) receiving the intervention in 2013.

\section{Nursing}

Improvements of between $6 \%$ and $62 \%$ were noted in delivery of patient education and $10 \%$ and $37 \%$ for carer education. Little overall change $(6 \%)$ was seen in patient education about the nature of stroke with $27 \%(95 \%$ CI $4 \%$ to $49 \%$ ) receiving education in 2008 and $33 \%$ (95\% CI $-8 \%$ to $75 \%$ ) in 2013. A large overall improvement $(62 \%)$ was seen in patient education about stroke impairments and management from $7 \%$ (95\% CI $-6 \%$ to $19 \%)$ to $69 \%$ (95\% CI $44 \%$ to $94 \%$ ).

\section{Medicine}

Results relating to screening for anxiety and depression and discussion regarding return work and driving were variable after the final audit. There was a drop in documented evidence of screening for anxiety and depression from $27 \%$ (95\% CI $5 \%$ to $49 \%$ ) in 2009 to $0 \%$ in 2013 . A large improvement $(25 \%)$ was noted in discussion around return to work from $0 \%$ up to $100 \%$.

\section{DISCUSSION}

This study investigated the use of a multifaceted tailored behaviour change intervention programme, including audit and feedback, education and training, use of prompts and cues and resources, to increase the proportion of patients with stroke receiving best practice screening, assessment and treatment. Practice improvements of up to $100 \%$ were achieved over a 4 -year period. The majority of practice areas improved by at least $10 \%$, which we consider to be clinically worthwhile.

Changes in screening, assessment and intervention: large changes were recorded in several practice areas including sensation screening $(+75 \%)$ and rehabilitation $(+100 \%)$; neglect screening $(+92 \%)$ and assessment $(100 \%)$. Several factors may have enabled changes in screening and assessment practices, compared with intervention. First, screening and assessment resources were often freely available online and easy to obtain, administer and implement, requiring no special training (e.g., the Catherine Bergego Scale for neglect screening). Second, access to free education and training by senior therapists helped to address the skills and knowledge barrier (e.g., visual scanning for neglect rehabilitation). Third, assessments and interventions that showed the greatest improvement required minimal or easily accessible equipment. For example, the Nottingham Sensory Assessment uses everyday objects such as a comb, scissors and coins, making implementation easier. Another interesting observation is that most areas showing the greatest improvement had very low compliance rates at baseline (mostly $0 \%$ ).

While some practice areas improved, others were resistant to change. There was a drop in compliance with anxiety and depression screening $(-27 \%)$, and little or no change was recorded in the delivery of education to patients with stroke regarding the nature of stroke $(6 \%)$ or notifying local doctors regarding return to driving $(0 \%)$. Some areas showed improvement after the second audit but by the third or fourth audits, there was a drop in compliance, for example, standardised aphasia assessment $(0 \%)$; and standardised sensation assessment $(0 \%)$. This drop in compliance has been observed in other stroke implementation research and is described as a 'decay effect'. ${ }^{30}$

While a process evaluation was not conducted concurrently, there are some factors which may explain the lack of behaviour change in some areas, or sustainability of change. First, while we did not formally monitor staffing levels during the study period, we observed some staff turnover across all disciplines. Despite the staffing changes, we were still able to achieve practice improvements. Second, the dose of behaviour change intervention delivered may have been too small to effect change (e.g., annual audit feedback and education and training primarily delivered in phase I). Third, we only explored and addressed local barriers once, in 2009. The barriers facing staff may have changed after 2009. In addition, staff and managers changed during the subsequent audit periods and review of perceived barriers may need to have been acknowledged and addressed. Lastly, a lack of familiarity or agreement with some of the recommended assessments or interventions may explain the lack of uptake among some disciplines and has been observed in other implementation trials. ${ }^{13}$

Strengths of this study include use of a systematic approach to design and implement a multifaceted tailored intervention which targeted identified barriers. Use of the TDF and BCW is another strength, providing a framework for selecting and matching interventions to key barriers for each discipline. This is the first study to use such an approach when targeting the behaviour of clinicians and stroke rehabilitation practice.

Study limitations include the use of medical record audits as a measure of practice. While audits are widely used in implementation research, written medical records may not always accurately represent actual practice (e.g., they may under-report the use of screening, assessment and intervention). An alternative to auditing is observation of practice, which is more resource intensive and costly. This was not considered feasible for this study. Other study limitations include the small number of files audited ( $\mathrm{n}=15$ per cycle), use of a single site and lack of a control or comparison site.

Advantages of a theory-informed behaviour change programme: while tailored behaviour change interventions continue to be used widely in implementation research to improve practice, the results of such studies are variable and the 
best approach is still not clear. ${ }^{1}$ This study used the TDF and BCW as frameworks to identify, address and match identified barriers to appropriate behaviour change interventions. This theory-informed process resulted in a multifaceted tailored intervention programme aimed at changing professions' and individual clinicians' behaviour and practice. The use of the TDF and BCW is a newer approach to designing tailored interventions in implementation research. Other studies have used this approach to increase implementation of an early mobilisation intervention in acute hospital setting, ${ }^{31}$ improve management of the risk of falls after hospital discharge, ${ }^{32}$ improve the management of blood pressure ${ }^{33}$ and improve management of medication in people with multiple morbidities. ${ }^{34}$

\section{CONCLUSIONS}

The use of a multifaceted tailored behaviour change intervention programme including audit, feedback and education improved some practices of allied health, nursing and medical professionals over a 4-year period. Overall, more patients received best practice stroke rehabilitation. The quality improvement process needs to continue to sustain the gains made. The stroke service planned to conduct their own audits periodically using the same number of files and select criteria. These audits will focus on monitoring improvements made in practice areas targeted as well as target areas in need of further improvement, for example, patient and carer education. Discussions are ongoing with stroke unit staff on other ways to make the implementation process sustainable into the future.

Acknowledgements The authors would like to thank the stroke unit staff for their participation and to Brad Lloyd who assisted with physiotherapy education sessions.

Contributors AV, AM and KS did the conception, design, analysis and interpretation of data.

Funding This study was supported by an infrastructure grant from the Ingham Institute for Applied Medical Research. The funder had no role in the design, conduct, analyses or reporting of the study or in the decision to submit the manuscript for publication.

Competing interests AM was supported between 2007 and 2011 by fellowships from the National Institute of Clinical Studies - National Health and Medical Research Council, the National Stroke Foundation, the University of Sydney, Royal Rehabilitation Centre Sydney.

Ethics approval This study was approved by the SWSLHD Human Research and Ethics Committee (HRECNo. QA 2008/046).

Provenance and peer review Not commissioned; internally peer reviewed.

Data sharing statement Full copies of the resources, that is, audit tool, audit reports and audit presentations are available by request to angela.vratsistas@ sydney.edu.au.

Open Access This is an Open Access article distributed in accordance with the Creative Commons Attribution Non Commercial (CC BY-NC 4.0) license, which permits others to distribute, remix, adapt, build upon this work non-commercially, and license their derivative works on different terms, provided the original work is properly cited and the use is non-commercial. See: http://creativecommons.org/ licenses/by-nc/4.0/

(c) Published by the BMJ Publishing Group Limited. For permission to use (where not already granted under a licence) please go to http://www.bmj.com/company/ products-services/rights-and-licensing/

\section{REFERENCES}

1. Baker R, Camosso-Stefinovic J, Gillies C, et al. Tailored interventions to address determinants of practice. Cochrane Database Syst Rev 2015:CD005470.

2. Giguère $A$, Légaré $F$, Grimshaw J, et al. Printed educational materials: effects on professional practice and healthcare outcomes. Cochrane Database Syst Rev 2012;10:CD004398.

3. O'Brien M, Rogers S, Jamtvedt G, et al. Educational outreach visits: effects on professional practice and health care outcomes (Review). 2007.

4. Forsetlund L, Bjørndal A, Rashidian A, et al. Continuing education meetings and workshops: effects on professional practice and health care outcomes. Cochrane Database Syst Rev 2009:CD003030.

5. Flodgren G, Parmelli E, Doumit G, et al. Local opinion leaders: effects on professional practice and health care outcomes. status and date: New search for studies and content updated (conclusions changed). 2011.

6. Ivers N, Jamtvedt G, Flottorp S, et al. Audit and feedback: effects on professional practice and healthcare outcomes. Cochrane Database Syst Rev 2012:CD000259.

7. Grimshaw JM, Thomas RE, MacLennan G, et al. Effectiveness and efficiency of guideline dissemination and implementation strategies. Health Technol Assess 2004;8:iii-iv.

8. Jamtvedt G, Young JM, Kristoffersen DT, et al. Audit and feedback: effects on professional practice and health care outcomes. Cochrane Database Syst Rev 2006;2:CD000259.

9. Intercollegiate Stroke Working Party. National sentinel stroke audit 2010 round 7. Public report for England, Wales and Northern Ireland. London: Royal College of Physicians of London, 2011.

10. Schouten LM, Hulscher ME, Akkermans R, et al. Factors that influence the stroke care team's effectiveness in reducing the length of hospital stay. Stroke 2008;39:2515-21.

11. Cadilhac DA, Pearce DC, Levi CR, et al. Improvements in the quality of care and health outcomes with new stroke care units following implementation of a clinician-led, health system redesign programme in New South Wales, Australia. Qual Saf Health Care 2008:17:329-33.

12. Abilleira S, Ribera A, Sánchez E, et al. The Second stroke audit of catalonia shows improvements in many, but not all quality indicators. Int J Stroke 2012;7:19-24.

13. Salbach NM, Wood-Dauphinee S, Desrosiers J, et al. Facilitated interprofessional implementation of a physical rehabilitation guideline for stroke in inpatient settings: process evaluation of a cluster randomized trial. Implement Sci 2017;12:100.

14. National Stroke Foundation. Clinical guidelines for stroke management. Melbourne: National Stroke Foundation, 2005.

15. National Stroke Foundation. Clinical guidelines for stroke management. Melbourne, Australia: National Stroke Foundation, 2010.

16. National Stroke Foundation. National stroke audit - rehabilitation services report. Melbourne, Australia: National Stroke Foundation, 2012.

17. National Stroke Foundation. National stroke audit - rehabilitation services. Melbourne, Australia: National Stroke Foundation, 2010.

18. National Stroke Foundation. National stroke audit acute services clinical audit report. Melbourne, Victoria: National Stroke Foundation, 2009.

19. Flottorp $\mathrm{SA}$, Oxman $A D$, Krause J, et al. A checklist for identifying determinants of practice: a systematic review and synthesis of frameworks and taxonomies of factors that prevent or enable improvements in healthcare professional practice. Implement Sci 2013;8:35.

20. McCluskey A, Vratsistas-Curto A, Schurr K. Barriers and enablers to implementing multiple stroke guideline recommendations: a qualitative study. BMC Health Serv Res 2013;13:323.

21. Hoffmann TC, Glasziou PP, Boutron I, et al. Better reporting of interventions: template for intervention description and replication (TIDieR) checklist and guide. BMJ 2014;348:g1687.

22. Ogrinc G, Davies L, Goodman D, et al. SQUIRE 2.0 (Standards for QUality Improvement Reporting Excellence): revised publication guidelines from a detailed consensus Procesp. J Contin Educ Nurs 2015;46:501-7.

23. Chan DK, Cordato D, O'Rourke F, et al. Comprehensive stroke units: a review of comparative evidence and experience. Int J Stroke 2013;8:260-4.

24. Intercollegiate Stroke Working Party. National clinical guideline for stroke. 3rd ed. London: Royal College of Physicians, 2008.

25. Lindsay P, Bayley M, Hellings C, et al. Canadian best practice recommendations for stroke care: Summary.Canadian Med AssoJ 2008. 
26. Scottish Intercollegiate Guidelines Network. Management of patients with stroke or TIA: assessment, investigation, immediate management and secondary prevention. A national clinical guideline. Scotland: NHS Quality Improvement Scotland, 2008.

27. National Institute for Health and Clinical Excellence. How to change practice: understand, identify and overcome barriers to change. London: National Institute for Health and Clinical Excellence, 2007.

28. Michie S, Johnston M, Abraham C, et al. Making psychological theory useful for implementing evidence based practice: a consensus approach. Qual Saf Health Care 2005;14:26-33.

29. Michie S, van Stralen MM, West R. The behaviour change wheel: a new method for characterising and designing behaviour change interventions. Implement Sci 2011;6:42.

30. Cadilhac DA, Andrew NE, Stroil Salama E, et al. Improving discharge care: the potential of a new organisational intervention to improve discharge after hospitalisation for acute stroke, a controlled beforeafter pilot study. BMJ Open 2017;7:e016010.
31. Moore JE, Mascarenhas A, Marquez C, et al. Mapping barriers and intervention activities to behaviour change theory for mobilization of vulnerable elders in ontario (MOVE ON), a multi-site implementation intervention in acute care hospitals. Implementation Science 2014;9:1.

32. Thomas S, Mackintosh S. Use of the theoretical domains framework to develop an intervention to improve physical therapist management of the risk of falls after discharge. Phys Ther 2014;94:1660-75.

33. Peiris D, Thompson SR, Beratarrechea A, et al. GACD Hypertension Research Programme, Writing GroupHypertension Research Programme members. Behaviour change strategies for reducing blood pressure-related disease burden: findings from a global implementation research programme. Implement Sci 2015;10:1.

34. Sinnott C, Mercer SW, Payne RA, et al. Improving medication management in multimorbidity: development of the multimorbiditY collaborative medication review And decision making (MY COMRADE) intervention using the behaviour change wheel. Implement Sci 2015;10:1. 